\title{
Role of Doppler indices in prediction of perinatal outcome in preeclampsia
}

\author{
Swapnali C. Kshirsagar ${ }^{1}$, Sneha S. Shirodkar ${ }^{1}$, Sunil J. Yadav ${ }^{1}$, Umesh M. Zile $^{2}$
}

\author{
${ }^{1}$ Department of Obstetrics and Gynecology, BYL Nair and Topiwala National Medical College, Maharashtra, India \\ ${ }^{2}$ Department of Paediatrics, Vasantrao Pawar Medical College, Nasik, Maharashtra, India
}

Received: 26 July 2016

Accepted: 26 August 2016

\author{
*Correspondence: \\ Dr. Swapnali C. Kshirsagar, \\ E-mail: swapn.splendid@gmail.com
}

Copyright: (c) the author(s), publisher and licensee Medip Academy. This is an open-access article distributed under the terms of the Creative Commons Attribution Non-Commercial License, which permits unrestricted non-commercial use, distribution, and reproduction in any medium, provided the original work is properly cited.

\begin{abstract}
Background: Preeclampsia is a major cause of maternal and perinatal mortality and morbidity worldwide, particularly in developing countries. The objective of the study was to study Doppler indices in pregnancies with preeclampsia and to correlate Doppler indices with perinatal outcome.

Methods: This was a prospective study conducted within a period of September 2012 to August 2014 where 100 singleton preeclampsia patients attending tertiary care hospital were recruited. These patients were followed by serial Doppler assessment and the result of the last Doppler examination within 10 days of delivery was considered in the subsequent correlation with perinatal outcomes. Perinatal outcome was studied under major and minor adverse outcome.

Results: In this study out of total population $54 \%$ were primigravida patients. $43.75 \%$ caesarean sections done for fetal distress with abnormal Doppler. Bilateral uterine artery diastolic notch and umbilical artery S/D ratio have better sensitivity $(72.55 \%)$ for prediction of minor adverse outcome while uterine artery diastolic notch has better sensitivity $(86.67 \%)$ for major adverse outcome. Umbilical artery and middle cerebral artery RI have better specificity and positive predictive value for prediction of both major and minor adverse outcome. Bilateral uterine artery notch and umbilical artery S/D ratio have better negative predictive value for prediction of both major and minor adverse outcome.

Conclusions: In this study it is concluded that Doppler analysis helps not only earlier detection of uteroplacental and fetoplacental changes associated with the disease but also help to take decision for early intervention. Doppler technology has provided the best opportunity for repetitive non-invasive haemodynamic monitoring in pregnancy for fetal well-being evaluation and predicting perinatal outcome.
\end{abstract}

Keywords: Preeclampsia, Doppler, Perinatal outcome

\section{INTRODUCTION}

Preeclampsia is a major cause of maternal and perinatal mortality and morbidity worldwide, particularly in developing countries. ${ }^{1}$ The most common complication of preeclampsia is intrauterine growth retardation. ${ }^{2}$ In preeclampsia there is insufficient invasion of maternal spiral arteries by the trophoblast early in gestation, so the transformation of these vessels from high volume nonresponsive vessels does not take place. ${ }^{3}$ The essence of a pregnancy to develop normally is that there should be adequate supply of nutrients and oxygen. The principle study lines are the uterine and umbilical arteries. The uteroplacental blood flow has been found to be decreased in hypertensive pregnancies. To avoid perinatal risk it is imperative that fetus should be monitored during pregnancy and ultrasonography is commonly done for the same, but Doppler is more beneficial to study blood flow in diseases wherein alteration in fetoplacental circulation is anticipated. ${ }^{4}$ 
Fetal Doppler ultrasound report using continuous wave assessment of umbilical artery flow was published in 1977 (Fitzgerald), with the same systems, in 1993 Campbell published assessment of uteroplacental circulation and that high resistant wave forms were obtained in preeclampsia. Subsequently studies were done with color Doppler and in many centres this has become important screening technique to predict perinatal outcome.

In obstetrics, assessment of clinically relevant alterations in maternal and fetal circulations with Doppler sonography is a complex and controversial issue. The vessels interrogated are often too small to measure accurately and too tortuous to determine the angle of insonation. Therefore 3 indices have been used by most investigators to quantitate velocities in systole relative to diastole. ${ }^{5}$ The major benefit to the clinician of using these indices is that they are all independent of the angle of insonation as cosine of angle of insonation in both numerator and denominator cancel out and do not require a measurement of the diameter of the vessel.

In clinical studies, vessels with relatively high diastolic flow velocities are believed to reflect a low downstream impedance to flow and those with low diastolic velocities reflect high impedance. In this way, all the most commonly used indices; S/D ratio, PI, RI provide a semi quantitative assessment of impedance in the vessels interrogated.

\section{METHODS}

Present prospective study was conducted within a period of September 2012 to August 2014 in our tertiary centre, in the department of obstetrics and gynaecology, using Toshiba XARIO color Doppler diagnostic system with curvilinear probe array with frequency $3.5 \mathrm{MHz}$.

A total of 100 singleton preeclampsia patients were constituted the study population. Singleton pregnancies with age up to 35 years, gestational age beyond 20 weeks complicated with preeclampsia were included in the study. Preeclampsia is defined as multisystem disorder of unknown aetiology characterized by development of hypertension to the extent of $140 / 90 \mathrm{mmHg}$ or more with proteinuria after $20^{\text {th }}$ weeks in a previously normotensive and non-proteinuric women. Gestational age determination was based on a best estimate from menstrual history, clinical gestational age or fetal biometry, preferably in the first or early second trimester.

Pregnancies with multiple gestations, complicated with medical disorders and intrauterine fetal demise were excluded from the study.

The following vessels were studied with patient in a recumbent position

- Maternal bilateral uterine arteries (BUtA).
- Fetal umbilical artery (UA).

- Fetal middle cerebral artery (MCA).

Pulsatility index (PI), resistance index (RI) and systolic/diastolic ratio of these arteries were obtained as soon as patient diagnosed with preeclampsia. These values were considered abnormal when they lie beyond the $5^{\text {th }}$ and $95^{\text {th }}$ percentile for gestational age. Similarly, presence of bilateral uterine artery notch and absence/reversal of end diastolic flow in umbilical artery were also noted.

The patients were followed by serial Doppler assessment and the result of the last Doppler examination within 10 days of delivery was considered in the subsequent correlation with perinatal outcomes. Perinatal outcome was studied under major and minor adverse outcome; major adverse outcomes were perinatal deaths, major complications like hypoxic ischemic encephalopathy, intraventricular haemorrhage, sepsis, necrotising enterocolitis, NICU care > 10 days and minor adverse outcomes were caesarean delivery for fetal distress, admission to NICU, preterm delivery.

Statistical analysis were made using statistical package for the social sciences software and qualitative data was presented with the help of frequency and percentage table. Sensitivity, specificity, positive and negative predictive values were calculated.

\section{RESULTS}

Preeclampsia is more prevalent in primigravidas. In total study population with respect to parity $54 \%$ of the women were primigravidas.

Table 1: Parity.

\begin{tabular}{|lll|}
\hline Parity & Frequency & Percent \\
\hline Primi & 54 & $54.00 \%$ \\
\hline G2 & 24 & $24.00 \%$ \\
\hline G3 and above & 22 & $22.00 \%$ \\
\hline Total & 100 & $100.00 \%$ \\
\hline Parity & Frequency & Percent \\
\hline Primi & 54 & $54.00 \%$ \\
\hline G2 & 24 & $24.00 \%$ \\
\hline G3 and above & 22 & $22.00 \%$ \\
\hline Total & 100 & $100.00 \%$ \\
\hline
\end{tabular}

Table 2: Mode of onset of labor.

\begin{tabular}{|lll|}
\hline $\begin{array}{l}\text { Mode of onset of labor } \\
\text { Caesarean section without trial } \\
\text { of labor }\end{array}$ & 29 & 29.00 \\
\hline Spontaneous & 23 & 23.00 \\
\hline Induction & 48 & 48.00 \\
\hline Total & $\mathbf{1 0 0}$ & $\mathbf{1 0 0 . 0 0}$ \\
\hline
\end{tabular}


Out of 100 patients $23 \%$ patients went in spontaneous labor while $48 \%$ needed induction and $29 \%$ had caesarean section without trial of labor for various indications. 21 patients needed caesarean section for the indication of fetal distress with abnormal Doppler findings while 18 were due to severe preeclampsia.

Table 3: Mode of delivery.

\begin{tabular}{|lll|}
\hline Mode of delivery & Frequency & Percent \\
\hline FTND & 42 & 42.00 \\
\hline PTD & 10 & 10.00 \\
\hline FT LSCS & 25 & 25.00 \\
\hline PT LSCS & 23 & 23.00 \\
\hline Total & $\mathbf{1 0 0}$ & $\mathbf{1 0 0 . 0 0}$ \\
\hline
\end{tabular}

Table 4: Birth weight.

\begin{tabular}{|lll|}
\hline Birth weight & Frequency & Percent \\
\hline ELBW & 8 & 8.00 \\
\hline VLBW & 10 & 10.00 \\
\hline LBW & 34 & 34.00 \\
\hline$>2.5 \mathrm{Kg}$ & 48 & 48.00 \\
\hline Total & $\mathbf{1 0 0}$ & $\mathbf{1 0 0 . 0 0}$ \\
\hline
\end{tabular}

Mean birth weight for present study is about $2.31 \mathrm{~kg}$ with standard deviation 0.647 . $8 \%$ babies had extremely low birth weight i.e. $<1 \mathrm{~kg}$, while $10 \%$ fall in very low birth weight category and 34\% fall under low birth weight i. e. $<2.5 \mathrm{~kg}$. Prematurity as well as intrauterine growth restriction both accounts for this.

Table 5: Perinatal outcome.

\begin{tabular}{|lll|}
\hline & Frequency & Percent \\
\hline Expired & 14 & 14.00 \\
\hline Alive & 86 & 86.00 \\
\hline Total & 100 & 100.00 \\
\hline
\end{tabular}

48 Babies had NICU admissions. 17 babies had NICU stay > 10 days and 28 babies had neonatal complications like hypoxic ischemic encephalopathy, neonatal enterocolitis, intraventricular or pulmonary haemorrhage. 13 babies were having poor APGAR $<7$ at 5 min. 36 babies had IUGR. 14 babies were expired. Poor neonatal outcome was strongly associated with absent or reversed end diastolic flow.

Out of 100 mothers 5 had episodes of convulsion, 9 had developed postpartum haemorrhage and one had evidence of acute renal failure. No maternal mortality was observed in present study.

Among study population $75 \%$ patients with abnormal PI had evidence of minor adverse outcome while, $50 \%$ patients had minor adverse outcome despite normal index. $50 \%$ patients with abnormal bilateral PI had major adverse outcome while, $29.2 \%$ had major adverse outcome despite no evidence of abnormal index in bilateral uterine artery.

$15.7 \%$ patients with abnormal umbilical artery resistance index had minor adverse outcome while $84.3 \%$ patients had it without evidence of abnormal resistance index. $23.3 \%$ patients with abnormal resistance index had major adverse outcome while $76.7 \%$ patients had major adverse outcome despite no evidence of abnormal resistance index.

Table 6 (a): Efficacy of bilateral uterine artery Doppler indices in predicting minor and major adverse outcome. Pulsatility index - minor adverse outcome.

\begin{tabular}{|llll|}
\hline \multirow{2}{*}{ BUtA PI } & \multicolumn{3}{l|}{ MAO } \\
\hline Abnormal & Count & 3 & No \\
\hline & Percent & $75.0 \%$ & 1 \\
\hline Normal & Count & 48 & $48.0 \%$ \\
\hline & Percent & $50.0 \%$ & $50.0 \%$ \\
\hline Total & Count & 51 & 49 \\
\hline & Percent & $51.0 \%$ & $49.0 \%$ \\
\hline Measure & Estimate & $95 \%$ confidence intervals \\
\cline { 2 - 4 } & Lower & Upper \\
\hline Sensitivity & $5.88 \%$ & 2.021 & 15.92 \\
\hline Specificity & $97.96 \%$ & 89.31 & 99.64 \\
\hline PPV & $75.00 \%$ & 30.06 & 95.44 \\
\hline NPV & $50.00 \%$ & 40.19 & 59.81 \\
\hline $\begin{array}{l}\text { Diagnostic } \\
\text { accuracy }\end{array}$ & $51.00 \%$ & 41.35 & 60.58 \\
\hline
\end{tabular}

Table 6 (b): Efficacy of bilateral uterine artery Doppler indices in predicting minor and major adverse outcome. Pulsatility index - major adverse outcome.

\begin{tabular}{|c|c|c|c|c|}
\hline \multirow{2}{*}{ BUtA PI } & \multicolumn{3}{|c|}{ MJAO } & \multirow{2}{*}{ Total } \\
\hline & & Yes & No & \\
\hline \multirow[t]{2}{*}{ Abnormal } & Count & 2 & 2 & 4 \\
\hline & Percent & $50.0 \%$ & $50.0 \%$ & $100.0 \%$ \\
\hline \multirow[t]{2}{*}{ Normal } & Count & 28 & 68 & 96 \\
\hline & Percent & $29.2 \%$ & $70.8 \%$ & $100.0 \%$ \\
\hline \multirow[t]{2}{*}{ Total } & Count & 30 & 70 & 100 \\
\hline & Percent & $30.0 \%$ & $70.0 \%$ & $100.0 \%$ \\
\hline \multirow{2}{*}{ Measure } & \multirow{2}{*}{ Estimate } & \multicolumn{3}{|c|}{$95 \%$ confidence intervals } \\
\hline & & Lower & \multicolumn{2}{|c|}{ Upper } \\
\hline Sensitivity & $6.67 \%$ & 1.848 & \multicolumn{2}{|c|}{21.32} \\
\hline Specificity & $97.14 \%$ & 90.17 & \multicolumn{2}{|c|}{99.21} \\
\hline PPV & $50.00 \%$ & 15 & \multicolumn{2}{|c|}{85} \\
\hline NPV & $70.83 \%$ & 61.08 & \multicolumn{2}{|c|}{78.98} \\
\hline $\begin{array}{l}\text { Diagnostic } \\
\text { Accuracy }\end{array}$ & $70.00 \%$ & 60.41 & \multicolumn{2}{|c|}{78.11} \\
\hline
\end{tabular}


Table 6 (c): Efficacy of bilateral uterine artery

Doppler indices in predicting minor and major adverse outcome. Resistance index - minor adverse outcome.

\begin{tabular}{|lllll|}
\hline UA RI & \multicolumn{5}{l|}{ MAO } & Total \\
\hline Abnormal & Count & 8 & 0 & 8 \\
\hline & Percent & $15.7 \%$ & $0.0 \%$ & $8.0 \%$ \\
\hline Normal & Count & 43 & 49 & 92 \\
\hline & Percent & $84.3 \%$ & $100.0 \%$ & $92.0 \%$ \\
\hline Total & Count & 51 & 49 & 100 \\
\hline & Percent & $100.0 \%$ & $100.0 \%$ & $100.0 \%$ \\
\hline Measure & Estimate & $95 \%$ Confidence & intervals \\
\cline { 3 - 6 } & Lower & \multicolumn{3}{l|}{ Upper } \\
\hline Sensitivity & $15.69 \%$ & 8.169 & \multicolumn{2}{c|}{28.01} \\
\hline Specificity & $100.00 \%$ & 92.73 & & 100 \\
\hline PPV & $100.00 \%$ & 67.56 & & 100 \\
\hline NPV & $53.26 \%$ & 43.14 & & 63.12 \\
\hline $\begin{array}{l}\text { Diagnostic } \\
\text { accuracy }\end{array}$ & $57.00 \%$ & 47.22 & & 66.27 \\
\hline
\end{tabular}

Table 6 (d): Efficacy of bilateral uterine artery Doppler indices in predicting minor and major adverse outcome. Resistance index - major adverse outcome.

\begin{tabular}{|c|c|c|c|c|}
\hline \multirow{2}{*}{ UA RI } & & \multicolumn{2}{|l|}{ MJAO } & \multirow{2}{*}{ Total } \\
\hline & & Yes & No & \\
\hline \multirow[t]{2}{*}{ Abnormal } & Count & 7 & 1 & 8 \\
\hline & Percent & $23.3 \%$ & $1.4 \%$ & $8.0 \%$ \\
\hline \multirow[t]{2}{*}{ Normal } & Count & 23 & 69 & 92 \\
\hline & Percent & $76.7 \%$ & $98.6 \%$ & $92.0 \%$ \\
\hline \multirow[t]{2}{*}{ Total } & Count & 30 & 70 & 100 \\
\hline & Percent & $100.0 \%$ & $100.0 \%$ & $100.0 \%$ \\
\hline \multirow{2}{*}{ Measure } & \multirow{2}{*}{ Estimate } & \multicolumn{3}{|c|}{ 95\% Confidence intervals } \\
\hline & & Lower & \multicolumn{2}{|c|}{ Upper } \\
\hline Sensitivity & $23.33 \%$ & 11.79 & \multicolumn{2}{|c|}{40.93} \\
\hline Specificity & $98.57 \%$ & 92.34 & \multicolumn{2}{|c|}{99.75} \\
\hline PPV & $87.50 \%$ & 52.91 & \multicolumn{2}{|c|}{97.76} \\
\hline NPV & $75.00 \%$ & 65.27 & \multicolumn{2}{|c|}{82.72} \\
\hline $\begin{array}{l}\text { Diagnostic } \\
\text { accuracy }\end{array}$ & $76.00 \%$ & 66.77 & \multicolumn{2}{|c|}{83.31} \\
\hline
\end{tabular}

$72.5 \%$ patients with abnormal umbilical artery S/D ratio had minor adverse outcome while $27.5 \%$ patients had it without evidence of abnormal S/D ratio. $80.00 \%$ patients with abnormal umbilical artery $\mathrm{S} / \mathrm{D}$ ratio had major adverse outcome while $20.00 \%$ patients had major adverse outcome despite no evidence of abnormal S/D ratio.

Among study population 45.1 patients with abnormal PI had evidence of minor adverse outcome while, $52.9 \%$ patients had minor adverse outcome despite normal index. $43.3 \%$ patients with abnormal middle cerebral artery PI had major adverse outcome while, 53.3\% had major adverse outcome despite no evidence of abnormal $\mathrm{PI}$ in middle cerebral artery.

Table 6 (e): Efficacy of bilateral uterine artery

Doppler indices in predicting minor and major adverse outcome. S/D ratio - minor adverse outcome.

\begin{tabular}{|lllll|}
\hline \multirow{2}{*}{ UA S/D } & & MAO & Total \\
\hline Abnormal & Count & 37 & 8 & 45 \\
\hline & Percent & $72.5 \%$ & $16.3 \%$ & $45.0 \%$ \\
\hline Normal & Count & 14 & 41 & 55 \\
\hline & Percent & $27.5 \%$ & $83.7 \%$ & $55.0 \%$ \\
\hline Total & Count & 51 & 49 & 100 \\
\hline & Percent & $100.0 \%$ & $100.0 \%$ & $100.0 \%$ \\
\hline Measure & Estimate & $95 \%$ Confidence intervals \\
\cline { 1 - 4 } & Lower & Upper \\
\hline Sensitivity & $72.55 \%$ & 59.05 & 82.89 \\
\hline Specificity & $83.67 \%$ & 70.96 & 91.49 \\
\hline PPV & $82.22 \%$ & 68.67 & 90.71 \\
\hline NPV & $74.55 \%$ & 61.7 & 84.19 \\
\hline $\begin{array}{l}\text { Diagnostic } \\
\text { accuracy }\end{array}$ & $78.00 \%$ & 68.93 & 85 \\
\hline
\end{tabular}

Table 6 (f): Efficacy of bilateral uterine artery

Doppler indices in predicting minor and major adverse outcome. S/D ratio - major adverse outcome.

\begin{tabular}{|lllll|}
\hline \multirow{2}{*}{ UA S/D } & & MJAO & & Total \\
\hline Abnormal & Count & 24 & 21 & 45 \\
\hline & Percent & $80.0 \%$ & $30.0 \%$ & $45.0 \%$ \\
\hline Normal & Count & 6 & 49 & 55 \\
\hline & Percent & $20.0 \%$ & $70.0 \%$ & $55.0 \%$ \\
\hline Total & Count & 30 & 70 & 100 \\
\hline & Percent & $100.0 \%$ & $100.0 \%$ & $100.0 \%$ \\
\hline Measure & Estimate & $95 \%$ Confidence intervals \\
\cline { 3 - 5 } & Lower & Upper \\
\hline Sensitivity & $80.00 \%$ & 62.69 & 90.5 & \\
\hline Specificity & $70.00 \%$ & 58.46 & 79.46 & \\
\hline PPV & $53.33 \%$ & 39.08 & 67.07 & \\
\hline NPV & $89.09 \%$ & 78.17 & 94.9 & \\
\hline $\begin{array}{l}\text { Diagnostic } \\
\text { accuracy }\end{array}$ & $73.00 \%$ & 63.57 & \multicolumn{2}{|c|}{80.73} \\
\hline
\end{tabular}

$19.6 \%$ patients with abnormal middle cerebral artery resistance index had minor adverse outcome while $80.4 \%$ patients had it without evidence of abnormal resistance index.

$26.7 \%$ patients with abnormal resistance index had major adverse outcome while $73.3 \%$ patients had major adverse outcome despite no evidence of abnormal resistance index. 
Table 7 (a): Efficacy of middle cerebral artery Doppler indices in predicting minor and major adverse outcome. Pulsatility index - minor adverse outcome.

\begin{tabular}{|lllll|}
\hline \multirow{2}{*}{ MCA PI } & \multicolumn{5}{l}{ MAO } & Total \\
\hline Abnormal & Count & 23 & 7 & 30 \\
\hline & Percent & $45.1 \%$ & $14.3 \%$ & $30.0 \%$ \\
\hline Normal & Count & 28 & 42 & 70 \\
\hline & Percent & $52.9 \%$ & $85.7 \%$ & $69.0 \%$ \\
\hline Total & Count & 51 & 49 & 100 \\
\hline & Percent & $100.0 \%$ & $100.0 \%$ & $100.0 \%$ \\
\hline Measure & Estimate & $95 \%$ Confidence intervals \\
\cline { 3 - 5 } & & Lower & Upper & \\
\hline Sensitivity & $45.10 \%$ & 32.27 & 58.62 & \\
\hline Specificity & $85.71 \%$ & 73.33 & 92.9 & \\
\hline PPV & $76.67 \%$ & 59.07 & 88.21 & \\
\hline NPV & $60.00 \%$ & 48.29 & 70.67 & \\
\hline $\begin{array}{l}\text { Diagnostic } \\
\text { Accuracy }\end{array}$ & $65.00 \%$ & 55.25 & 73.64 & \\
\hline & & \multicolumn{4}{|c|}{} \\
\hline
\end{tabular}

In uterine artery presence of notch and umbilical artery S/D Ratio has got maximum sensitivity and negative predictive value while umbilical artery and middle cerebral artery resistance index has got maximum specificity and positive predictive value for minor adverse outcome (Figure 1).

In uterine artery presence of notch has got maximum sensitivity and negative predictive value while umbilical artery resistance index has got maximum specificity and positive predictive value for major adverse outcome (Figure 2).

Table 7 (b): Efficacy of middle cerebral artery

Doppler indices in predicting minor and major adverse outcome. Pulsatility index - major adverse outcome.

\begin{tabular}{|lllll|}
\hline MCA PI & \multicolumn{5}{l}{ MJAO } & Total \\
\hline Abnormal & Count & 13 & 17 & 30 \\
\hline & Percent & $43.3 \%$ & $24.3 \%$ & $30.0 \%$ \\
\hline Normal & Count & 17 & 53 & 70 \\
\hline & Percent & $53.3 \%$ & $75.7 \%$ & $69.0 \%$ \\
\hline Total & Count & 30 & 70 & 100 \\
\hline & Percent & $100.0 \%$ & $100.0 \%$ & $100.0 \%$ \\
\hline Measure & Estimate & $95 \%$ Confidence intervals \\
\hline Sensitivity & $43.33 \%$ & 27.38 & 60.8 & \\
\hline Specificity & $75.71 \%$ & 64.5 & 84.25 & \\
\hline PPV & $43.33 \%$ & 27.38 & 60.8 & \\
\hline NPV & $75.71 \%$ & 64.5 & 84.25 & \\
\hline $\begin{array}{l}\text { Diagnostic } \\
\text { accuracy }\end{array}$ & $66.00 \%$ & 56.28 & 74.54 & \\
\hline
\end{tabular}

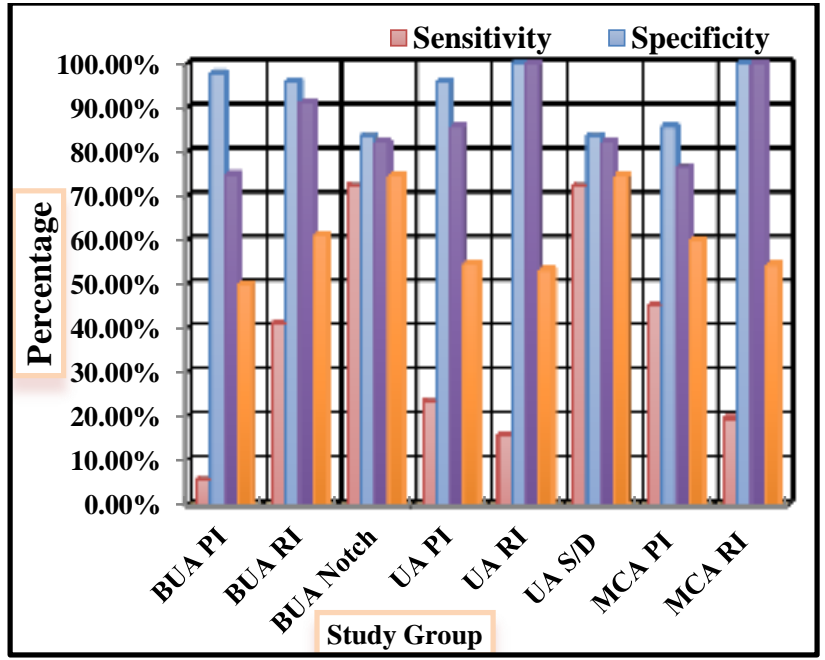

Figure 1: Comparison among study groups.

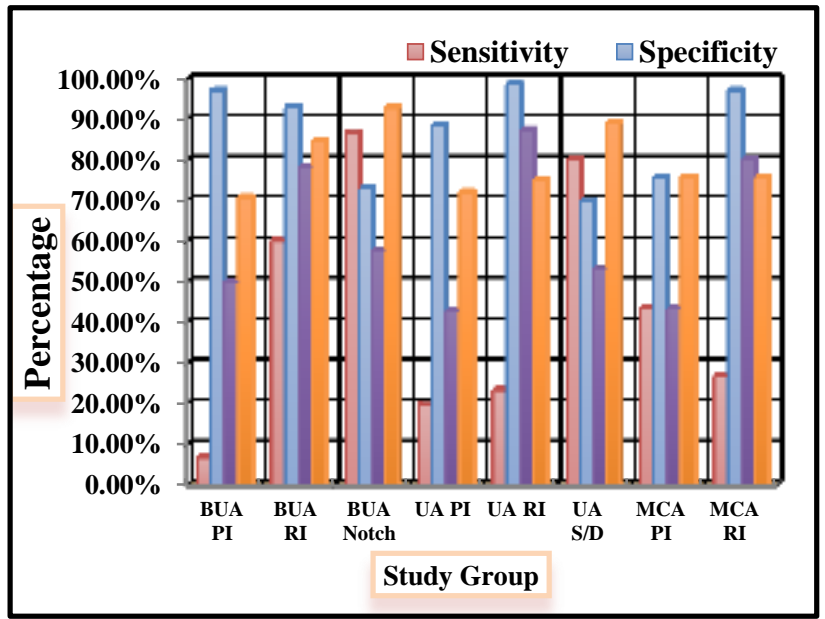

Figure 2: Comparison for MJO among study groups.

Table 7 (c): Efficacy of middle cerebral artery

Doppler indices in predicting minor and major adverse outcome. Resistance index - minor adverse outcome.

\begin{tabular}{|c|c|c|c|c|}
\hline \multirow{2}{*}{ MCA RI } & & \multicolumn{2}{|l|}{ MAO } & \multirow{2}{*}{ Total } \\
\hline & & Yes & No & \\
\hline \multirow[t]{2}{*}{ Abnormal } & Count & 10 & 0 & 10 \\
\hline & Percent & $19.6 \%$ & $0.0 \%$ & $10.0 \%$ \\
\hline \multirow[t]{2}{*}{ Normal } & Count & 41 & 49 & 90 \\
\hline & Percent & $80.4 \%$ & $100.0 \%$ & $90.0 \%$ \\
\hline \multirow[t]{2}{*}{ Total } & Count & 51 & 49 & 100 \\
\hline & Percent & $100.0 \%$ & $100.0 \%$ & $100.0 \%$ \\
\hline \multirow{2}{*}{ Measure } & \multirow{2}{*}{ Estimate } & \multicolumn{3}{|c|}{ 95\% Confidence intervals } \\
\hline & & Lower & \multicolumn{2}{|c|}{ Upper } \\
\hline Sensitivity & $19.61 \%$ & 11.02 & \multicolumn{2}{|c|}{32.46} \\
\hline Specificity & $100.00 \%$ & 92.73 & \multicolumn{2}{|l|}{100} \\
\hline PPV & $100.00 \%$ & 72.25 & \multicolumn{2}{|l|}{100} \\
\hline NPV & $54.44 \%$ & 44.18 & \multicolumn{2}{|l|}{64.34} \\
\hline $\begin{array}{l}\text { Diagnostic } \\
\text { accuracy }\end{array}$ & $59.00 \%$ & 49.2 & \multicolumn{2}{|l|}{68.13} \\
\hline
\end{tabular}


Table 7 (d): Efficacy of middle cerebral artery

Doppler indices in predicting minor and major adverse outcome. Resistance index - major adverse outcome.

\begin{tabular}{|lllll|}
\hline MCA RI & \multicolumn{5}{l|}{ MJAO } & Total \\
\hline Abnormal & Count & 8 & 2 & 10 \\
\hline & Percent & $26.7 \%$ & $2.9 \%$ & $10.0 \%$ \\
\hline Normal & Count & 22 & 68 & 90 \\
\hline & Percent & $73.3 \%$ & $97.1 \%$ & $90.0 \%$ \\
\hline Total & Count & 30 & 70 & 100 \\
\hline & Percent & $100.0 \%$ & $100.0 \%$ & $100.0 \%$ \\
\hline Measure & Estimate & $95 \%$ Confidence intervals \\
\cline { 3 - 5 } & Lower & Upper & \\
\hline Sensitivity & $26.67 \%$ & 14.18 & 44.45 & \\
\hline Specificity & $97.14 \%$ & 90.17 & 99.21 & \\
\hline PPV & $80.00 \%$ & 49.02 & 94.33 & \\
\hline NPV & $75.56 \%$ & 65.75 & 83.27 & \\
\hline $\begin{array}{l}\text { Diagnostic } \\
\text { accuracy }\end{array}$ & $76.00 \%$ & 66.77 & 83.31 & \\
\hline
\end{tabular}

\section{DISCUSSION}

Preeclampsia is strongly related to development and function of uteroplacental and fetoplacental circulations. This results in improper blood flow in uterine and umbilical arteries. An adequate fetal circulation is necessary for normal fetal growth. To facilitate this, remarkable maternal and placental vasculature changes occur during pregnancy.

\section{Uterine artery}

Hemodynamic changes occurring on the maternal side of placenta is reflected in uterine artery by 20 weeks of gestation. Trophoblastic cells penetrate the maternal spiral artery within inner $1 / 3 \mathrm{rd}$ of myometrium disintegrating the internal elastic lamina of spiral arteries by 25 weeks. This results in maximum dilatation and minimum vascular resistance in the vessel. ${ }^{6}$ In preeclampsia there is inadequate invasion leading to increased resistance in spiral arteries. This leads to impedance of blood flow in uterine arteries. ${ }^{7}$ The S/D ratio in uterine artery greater than 2.6 is considered abnormal. The presence of early diastolic notch is normal phenomenon up to 26 weeks of gestation. Presence of notch after 26 weeks is a bad indicator suggesting increased impedance of blood flow. ${ }^{8}$

Fichera et al studied 100 consecutive singleton pregnancies complicated with preeclampsia and mean uterine artery PI values obtained at the time of diagnosis of preeclampsia were analysed. Their data confirm that mean uterine artery PI, assessed at diagnosis of preeclampsia, represent a good independent predictor for gestational age at delivery and birth weight percentile. However the predictive value for the subsequent development of adverse perinatal outcome does not seem to be clinically relevant. ${ }^{9}$ In our study we observed that bilateral uterine artery pulsatility index has got maximum specificity for both major and minor adverse outcome among other uterine artery indices.

Asselt KV et al studied one hundred and eight pregnant women with pre-eclampsia were followed and examined by Doppler velocimetry of the uterine arteries. The presence of a notch and the mean pulsatility index (PI) in the two arteries were recorded. The combination of umbilical and uterine artery waveform results was the best predictor of adverse outcome. ${ }^{10}$ In our study we observed that presence of notch has got maximum sensitivity and negative predictive value for both major and minor adverse outcome. In our study by applying Chi-square test we got $\mathrm{P}$-value significant for all uterine artery indices except for pulsatility index.

\section{Umbilical artery Doppler}

Umbilical artery velocimetry correlates with hemodynamic changes in the fetoplacental circulation. With increase in number of tertiary stem villi and arterial channels, fetoplacental compartment develops and the impedance in the umbilical artery decreases. From 15 weeks of gestation umbilical artery resistance declines and the diastolic component appears in the waveform during early second trimester. ${ }^{11} \mathrm{~S} / \mathrm{D}$ ratio of less than or equal to 3 is considered normal.

Alexander et al studied 68 pregnant women with hypertension and concluded that umbilical artery S/D ratio alone is better predictor of IUGR and poor pregnancy outcome than the uterine artery S/D ratio. ${ }^{12}$ In our study umbilical artery S/D ratio has got maximum sensitivity and negative predictive value for both major and minor adverse outcome among other umbilical artery indices.

Borges KJJ et al studied ninety pregnant women with pregnancy induced hypertension with gestational age greater than 35weeks. Doppler ultrasound examinations were carried out to record umbilical artery resistive index (UA RI). They found that increasing resistance in umbilical arteries leads to, decreased placental thickness and placental weight, earlier placental maturity, greater number of placental infarcts, decreased amniotic fluid production, and lower birth weight. ${ }^{13}$ In our study we observed that umbilical artery resistance index has got maximum specificity and positive predictive value for both major and minor adverse outcome among other umbilical artery indices. In our study by applying Chisquare test we got $\mathrm{P}$ value significant for all indices of umbilical artery.

\section{Middle cerebral artery}

In normal fetus, there is little diastolic flow in MCA and $\mathrm{S} / \mathrm{D}$ ratio is greater than 4 . In asymmetric IUGR there is 
increased diastolic flow, a pattern believed to reflect brain sparing phenomena described in experimental models of fetal hypoxia.

Lakhkar BN et al studied fifty-eight singleton pregnancies beyond 30 weeks of gestation complicated by intrauterine growth restriction and severe preeclampsia or both were prospectively examined with Doppler US of the UA, MCA, UV and IVC and found that there were a total of 12 perinatal deaths in study group. Of these seven were neonatal deaths (NND) and five were stillbirths. MCA pulsatility index (P.I) is the most specific index $(90.9 \%)$ for predicting in any adverse perinatal outcome while umbilical artery $\mathrm{S} / \mathrm{D}$ ratio is the most sensitive index $(66.6 \%)$ in predicting any adverse perinatal outcome i.e. including both major and minor outcome. $^{14}$

In present study, it was found that middle cerebral artery pulsatility index has maximum sensitivity and negative predictive value for both major and minor adverse outcome while resistance index has maximum specificity and positive predictive value for both major and minor adverse outcome. By applying chi-square test we got significant $\mathrm{P}$-value for all indices of middle cerebral artery studied here.

\section{CONCLUSION}

- Preeclampsia during pregnancy is the most common medical condition encountered. Doppler analysis helps not only earlier detection of uteroplacental and fetoplacental changes associated with the disease but also help to take decision for early intervention. Intervention may require to be done in preterm gestation so patient can be referred to higher centre for better NICU facilities which can be decided soon because of abnormal Doppler changes. In our study out of 33 preterm deliveries 24 were referred patients. 12 preterm babies expired while 21 babies survived in our institution.

- Presence of diastolic notch is very reliable criteria to predict future outcome of the fetus. Umbilical artery Doppler has got better sensitivity and predictive value for fetal adverse outcome. Abnormal fetoplacental circulation is more associated with adverse outcome as compared to abnormal uteroplacental circulation.

- Umbilical artery Doppler findings are slight better predictor of adverse perinatal outcome than an abnormal MCA. Thus Doppler ultrasound makes it possible to distinguish between a compensated and decompensated placental insufficiency.

- Doppler technology has provided the best opportunity for repetitive non-invasive haemodynamic monitoring in human pregnancy.

- Perinatal morbidity and mortality can be reduced by fetal surveillance with Doppler velocimetry study. In this small and preliminary study we have assessed the role of Doppler ultrasound and found it to be a useful technique for fetal well-being evaluation and predicting perinatal outcome.

\section{ACKNOWLEDGEMENTS}

Authors would like to thank head of unit department of obstetrics and gynecology Dr. Asha Dalal, Nair Hospital, Mumbai, for her constant support and encouragement to accomplish this study. Also authors are thankful to Ethics committee for academic research projects (ECARP) for approving this study. The authors would also like to thank all the patients who had participated in the study.

Funding: No funding sources

Conflict of interest: None declared

Ethical approval: The study was approved by the Institutional Ethics Committee

\section{REFERENCES}

1. Villar J, Say L, Gulmezoglu M. Eclampsia and preeclampsia: a worldwide health problem for 2000 years. In :Critchley, Mac Lean A, Poston L, Walker J(Eds). Preeclampsia. Landon: RCOG Press. 2003;189-207.

2. Khalid M, Wahab S, Kumar V, Khalid S, Haroon S, Noor A. Sabzposh Doppler indices in prediction of fetal outcome in hypertensive pregnant women. Nepal Journal of Obstetrics and Gynaecology. 2011;6(1):28-34.

3. Roberts JM, Cooper DW. Pathogenesis and Genetics of preeclampsia (review). Lancet. 2001;357:53-6.

4. Bano S, Chaudhary V, Pandey S, Mehta UL, Sharma AK. Color Doppler evaluation of cerebral umbilical pulsatility ratio and its usefulness in the diagnosis of intrauterine growth retardation and prediction of adverse perinatal outcome. 2010;20(1):20-5.

5. Fleischer, Ruth B, Goldstein. Collen ultrasonography in obstetrics and gynecology; 2010:53-54.

6. Maler J, Manor D, Itskovitz J. Changes in uterine blood flow during human pregnancy. Am J Obstet Gynecol. 1990;162:121-5.

7. Maulik D, Yarlagadda P, Downing G. Doppler velocimetry in obstetrics. Obstet Gynecol Clin North Am. 1990;17:63-86.

8. Park YW, Choe JS, Kim HS, Kim JS. The clinical implication of early diastolic notch in third trimester Doppler analysis of uterine artery J. Ultrasound Med. 1996;15:47-51.

9. Fichera A, Pagani G, Gerosa V, Gregorini M, Rovida $\mathrm{P}$, Prefumo F, et al. The role of uterine artery pulsatility index for prediction of outcome in pregnancies complicated by pre-eclampsia. Ultrasound in Obstet Gynae. 2012;40(1):171-310.

10. Asselt KV, Gudmundsson S, Lindqvist P, Marsal K. Uterine and umbilical artery velocimetry in preeclampsia. 1998;77(6):614-5.

11. Schulman H, Gleischer A, Stern W. Umbilical wave ratios in human pregnancy. Am J Obstet Gynecol. 1984;148:985-90. 
12. Kofinas AD, Penry M, Nelson LH, Meis PJ, Swain M, Salem W. Uterine and umbilical artery flow velocity waveform analysis in pregnancies complicated by chronic hypertension or preeclampsia. Southern Medical Journal. 1990;83:2.

13. Borges KJJ, Hassan N, Hussain R, Akhtar MT. Effects of variation in umbilical artery resistive index on placental morphology and birth weight in pregnancy induced hypertension. J Ayub Med Coll Abbottabad. 2013;25(3):23-6.

14. Lakhkar BN, Rajagopal KV, Gourisankar PT. Doppler prediction of adverse perinatal outcome in $\mathrm{PIH}$ and IUGR. Indian $\mathrm{J}$ Radiol Imaging. 2006;16:109-16.

Cite this article as: Kshirsagar SC, Shirodkar SS, Yadav SJ, Zile UM. Role of Doppler Indices in prediction of perinatal outcome in preeclampsia. Int $\mathbf{J}$ Reprod Contracept Obstet Gynecol 2016;5:3390-7. 